\title{
Compresibilidad y propiedades dinámicas de suelos diatomáceos de Mejillones
}

\section{Compressibility and dynamic properties of diatomaceous soils of Mejillones}

Fecha de entrega: 5 de diciembre 2018

Fecha de aceptación: 1 de marzo 2019

\section{Ghio Arenaldi ${ }^{1}$, Carlos Ovalle ${ }^{2}$ y Antonio Barrios ${ }^{3}$}

${ }^{1}$ Departamento de Ingeniería Estructural y Geotécnica, Pontificia Universidad Católica de Chile, Avda. Vicuña Mackenna 4860, Santiago, 7820436, Chile, garenaldi@uc.cl

${ }^{2}$ Research Institute of Mining and Environment RIME UQAT-Polytechnique, Department of Civil, Geological and Mining Engineering, Polytecnique Montréal, Room A-356, 2900 Édouard Montpetit Blvd, Montreal, QC, H3T 1J4, Canada, carlos.ovalle@polymtl.ca

${ }^{3}$ Petrus Consultores Geotécnicos Ltda., El Alba 2 S/No, Parcela 39, Lote 11, Loteo Los Nogales de Chicureo, Colina, Chile, abarrios@petrus.cl

En este artículo se presenta un exhaustivo estudio de laboratorio sobre compresibilidad primaria y secundaria de un suelo diatomáceo de la Bahía de Mejillones, en el norte de Chile. Se reportan observaciones microscópicas SEM de los fósiles de diatomeas y de su composición mineralógica. A pesar de su baja densidad y alta plasticidad, el material alcanza altos valores de presión de preconsolidación. Sobre dicha presión, el suelo es altamente compresible, presumiblemente debido a la rotura de frústulas de diatomeas, y presenta significativas deformaciones por creep. También se reportan propiedades dinámicas y curvas de degradación de módulo de corte, donde los resultados de $G_{\max }$ son consistentes con los valores de $V_{s}$ medidos in-situ.

Palabras clave: diatomeas, propiedades indice, compresibilidad, creep, microestructura, degradación del módulo de corte
This article presents a comprehensive laboratory study on primary and secondary compressibility of a diatomaceous soil from Mejillones Bay in northern Chile. SEM microscopic observations of the diatom fossils and their mineralogical composition are reported. Despite its low density and high plasticity, the material reaches a high overconsolidation pressure. Over that pressure, the soil is highly compressible, presumably due to the breakage of diatoms frustules, and presents significant creep strains. Dynamic properties and shear modulus degradation curves are also reported, where the results of $G_{\max }$ are consistent with values $V_{s}$ of measured in-situ. Keywords: diatoms, index properties, compressibility, creep, microstructure, shear modulus degradation

\section{Introducción}

La bahía de Mejillones, ubicada en la Región de Antofagasta en el norte de Chile, ha experimentado un importante desarrollo industrial y portuario en los últimos 20 años, principalmente para prestar servicios a las industrias de la minería y energía (Figura 1). Gran parte de estas obras están fundadas en suelos diatomáceos, compuestos por una mezcla de arenas, finos y fósiles de diatomeas. Esta composición entrega al suelo un comportamiento geotécnico singular que no coincide con correlaciones clásicas usualmente aplicadas en Ingeniería Geotécnica. Además, la información reportada sobre el comportamiento del material inalterado es escasa.

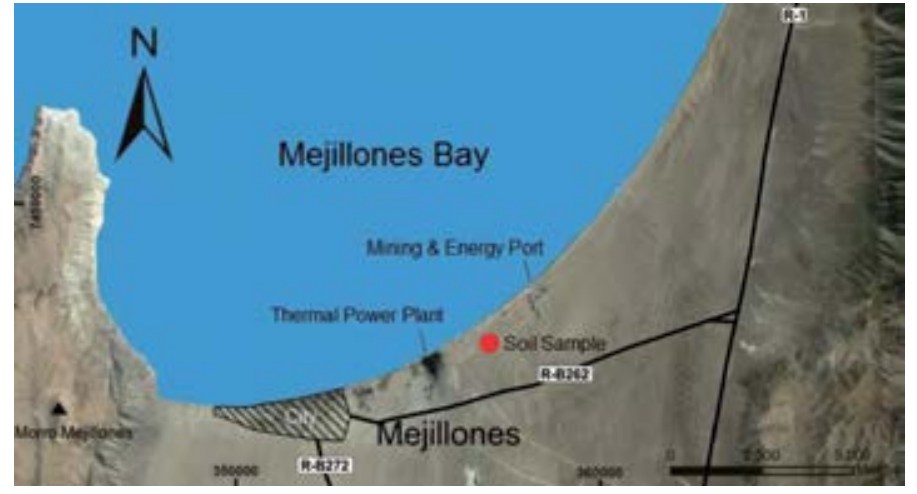

Figura 1: Bahía de Mejillones (modificado de González, 2013)

Las diatomeas son microalgas unicelulares que constituyen uno de los tipos más comunes de fitoplancton. Su tamaño varía de 10 a $100 \mu \mathrm{m}$ y poseen una frústula externa dura y 
porosa compuesta casi en su totalidad de sílice ( D í a z Rodríguez y González-Rodríguez, 2013). En general, la fuente de sílice proviene de actividad volcánica, lo que explica la presencia de estos suelos en sectores contiguos al cinturón de fuego del Pacífico; por ejemplo, Chile, Colombia, México y Japón (Arenaldi, 2018; Caicedo et al., 2018; Díaz-Rodríguez y González-Rodríguez, 2013; Hong et al., 2006; Sánchez, 2002; Verdugo, 2008; Wesley, 2010). Después de su muerte y descomposición orgánica, las frústulas se depositan al fondo de los océanos o lagos formando depósitos de suelos diatomáceos (Treguer et al., 1995). Debido a la estructura interna de los fósiles, estos suelos presentan baja densidad seca, altos contenidos de humedad y Límites de Atterberg que generalmente implican una clasificación de limo de alta plasticidad (Díaz-Rodríguez y González-Rodríguez, 2013; Nazar et al., 2016; Shiwakoti et al., 2002). Sin embargo, el suelo presenta una alta resistencia al corte y una presión de preconsolidación relativamente alta (Arenaldi, 2018; Hong et al., 2006; Sánchez, 2002; ; Nazar et al., 2016).

Diversos estudios han mostrado que el contenido de diatomeas en mezclas remoldeadas con Caolinita afecta los Límites de Atterberg (Díaz-Rodríguez y GonzálezRodríguez, 2013; Nava, 2007; Nazar et al., 2016; ). En la Figura 2 se aprecia la variación de la ubicación de mezclas de Diatomeas y Caolinita (D: K) en la carta de plasticidad de Casagrande, desde $\mathrm{CH}$ (Caolinita sobre la línea A) hacia $\mathrm{MH}$ al aumentar el contenido de diatomeas. Shiwakoti et al. (2002) agregan que a altos porcentajes de diatomeas y pese al alto contenido de humedad, el material se comporta como no plástico puesto que el agua queda atrapada en los fósiles y no tiene efecto en las fuerzas físico-químicas de interacción entre partículas. En otras palabras, el incremento en el contenido de diatomitas implica que el Límite Líquido aumente por efecto del agua atrapada en las frústulas, pero al mismo tiempo la plasticidad disminuye debido a que las diatomitas no representan un aporte en minerales arcillosos.

En este artículo se presenta un exhaustivo estudio de laboratorio sobre compresibilidad (primaria y secundaria) y propiedades dinámicas de muestras inalteradas de un suelo diatomáceo de la Bahía de Mejillones. También se analiza la microestructura del suelo y su mineralogía.

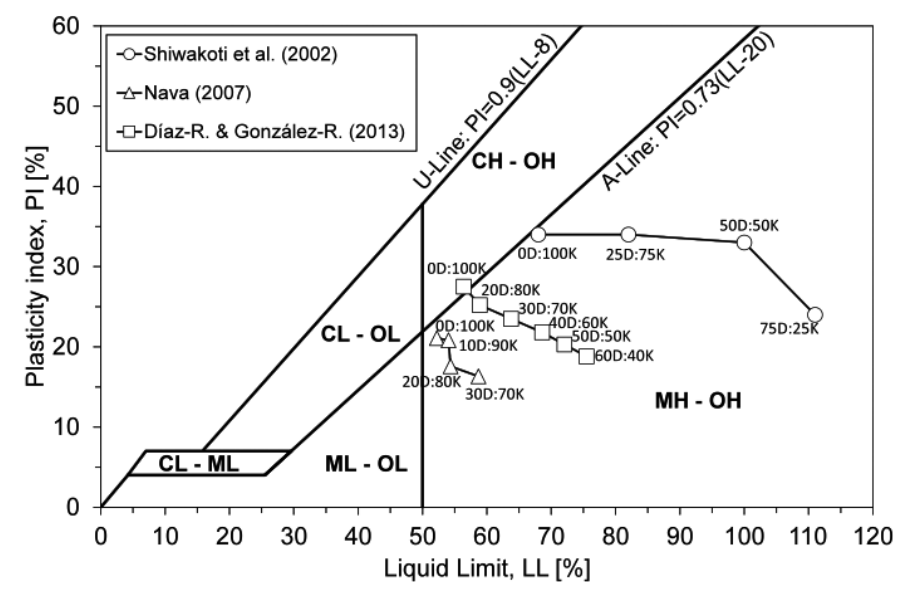

Figura 2: Evolución del material frente al contenido de Diatomita: Caolinita ( $\% \mathrm{D}: \% \mathrm{~K})$ en peso seco

\section{Sector de estudio}

El sector de estudio corresponde a la península de Mejillones, formado por un relieve morfológico originado por la acreción de rocas Paleozoicas (570 millones de años) al continente, donde las rocas conformantes son predominantemente del Jurásico (200-145 Ma) y Cretácico (145-65 Ma) según Muñoz y Rivas (2012) y Maringue (2017). Dentro de las principales unidades geológicas que afloran en esta península, los sedimentos que rellenan la cuenca de Mejillones corresponden a depósitos Cenozoicos relacionados con eventos del Neógeno (25 a $2 \mathrm{Ma}$ ), y a depósitos del Cuaternario recientes, correspondientes a arenas, limos, coquinas y diatomitas, en lo que se conoce como formación La Portada (González, 2013; Maringue, 2017; Muñoz y Rivas, 2012). Una revisión acerca de la génesis de los suelos diatomáceos en la zona se puede encontrar en Arenaldi (2018).

\section{Material de ensayo}

Se obtuvieron varias muestras en una misma calicata en la bahía de Mejillones, en la forma de bloques no alterados tallados a una profundidad de $3 \mathrm{~m}$ (Figura 1). El material fue sellado con película plástica, trasladado al laboratorio y almacenado en una cámara húmeda. Para caracterizar el suelo se realizaron ensayos de granulometría, densidad de sólidos, límites de consistencia (método húmedo), ensayo Proctor Estándar (método húmedo), visualización del material en microscopio electrónico de barrido SEM, espectroscopía SEM y ensayo de difracción de rayos $\mathrm{X}$ (XRD). En la Figura 3 se presentan las imágenes 
de microscopio SEM. El análisis XRD mostrado en la Figura 4 indica que la presencia de sílice en la forma de cuarzo $\left(\mathrm{SiO}_{2}\right)$ es predominante en el material, seguida por calcita $\left(\mathrm{CaCO}_{3}\right)$; esto es consistente con el ensayo de espectroscopia SEM (ver detalles en Arenaldi, 2018). Los análisis XRD y SEM no sugieren la presencia de minerales arcillosos en las muestras ensayadas. Las propiedades que caracterizan el suelo diatomáceo se presentan en la Tabla 1.

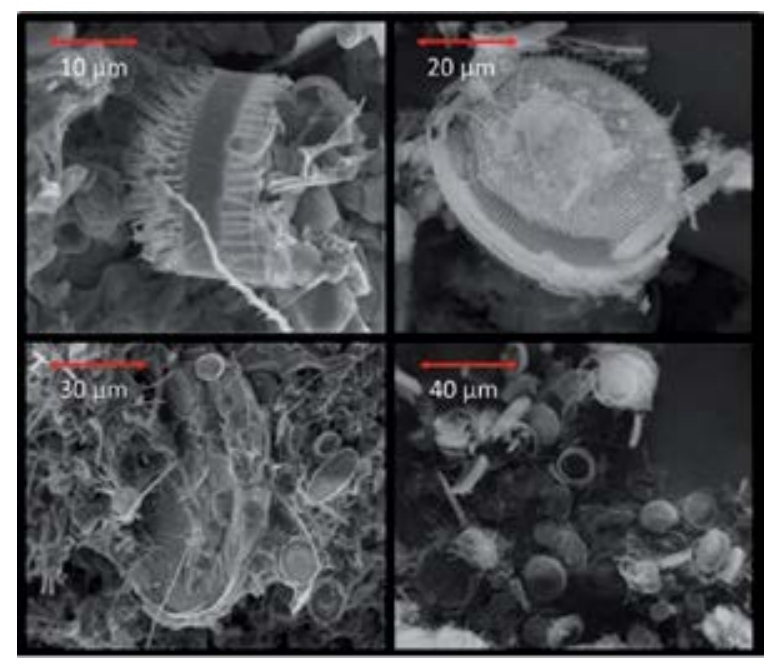

Figura 3: Microscopía SEM en muestras naturales

Tabla 1: Caracterización geotécnica

\begin{tabular}{|l|c|}
\hline \multicolumn{1}{|c|}{ Propiedades } & Resultados \\
\hline Límite Líquido LL, \% & 82 \\
\hline Índice de Plasticidad IP, \% & 38 \\
\hline Clasificación USCS & $\mathrm{MH}$ \\
\hline $\begin{array}{l}\text { Porcentaje de finos que pasa malla } \\
\# 200\end{array}$ & 72 \\
\hline Gravedad específica, $G_{\mathrm{s}}$ & 2.635 \\
\hline
\end{tabular}

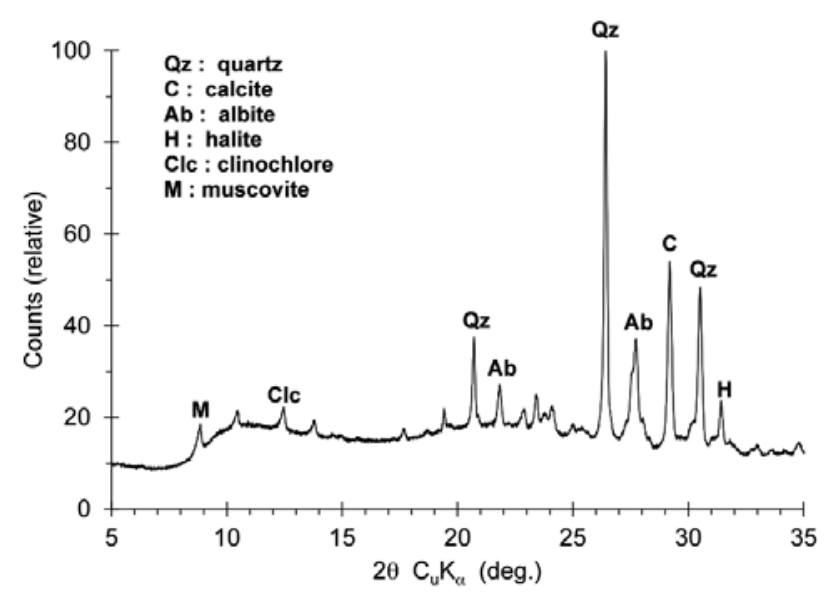

Figura 4: Análisis de difracción de rayos X en muestra de suelo diatomáceo

\section{Consolidación primaria}

Se efectuó una serie de ensayos edométricos en base a la norma ASTM D2435 (2011). Para ello, se utilizaron muestras inalteradas y remoldeadas de $50 \mathrm{~mm}$ de diámetro y $16 \mathrm{~mm}$ de altura. Las muestras remoldeadas fueron preparadas a distintas densidades y humedades para abarcar índices de vacíos distintos y confeccionadas mediante la compactación de 3 capas de suelo de una misma altura. La saturación por inundación se efectuó posterior a la estabilización de una carga de $98 \mathrm{kPa}$; no se observó hinchamiento ni colapso después de la inundación bajo carga constante. También se realizaron pruebas de consolidación isotrópica en muestras saturadas en base a ASTM D4767 (2011), con la fase de consolidación drenada en cámara triaxial aplicando contrapresión constante; utilizando muestras cilíndricas inalteradas de $50 \mathrm{~mm}$ de diámetro y 100 $\mathrm{mm}$ de altura. La saturación en las pruebas isotrópicas se verificó asegurando un parámetro de Skempton $B>0.95$.

Tabla 2: Resultados de muestras ensayadas

\begin{tabular}{|c|c|c|c|c|c|c|c|c|}
\hline Muestra & Notación & $\begin{array}{c}\gamma_{\mathrm{d}}, \\
\mathrm{kN} / \mathrm{m}^{3} \\
\end{array}$ & $\begin{array}{c}\text { Humedad } \\
w, \%\end{array}$ & $e_{0}$ & $\begin{array}{l}\sigma_{\mathrm{c}}^{\prime}, \\
\mathrm{kPa}\end{array}$ & $C_{c}{ }^{3}$ & $C_{s}$ & $C_{c} / C_{s}$ \\
\hline \multirow{5}{*}{$\begin{array}{c}\text { Inalteradas, } \\
\text { contenido } \\
\text { de humedad } \\
\text { natural }\end{array}$} & $\mathrm{N} 1$ & 7.46 & 91 & 2.46 & - & - & $0.083^{1}$ & - \\
\hline & $\mathrm{N} 2$ & 7.36 & 91 & 2.48 & . & - & $0.101^{1}$ & - \\
\hline & N3 & 6.38 & 96 & 3.01 & 450 & 1.63 & $0.104^{1}$ & 15.8 \\
\hline & $\mathrm{N} 4$ & 6.18 & 83 & 2.44 & 620 & 1.77 & $0.117^{1}$ & 15.0 \\
\hline & N5 & 6.57 & 93 & 2.91 & 450 & 1.63 & $0.120^{2}$ & 13.6 \\
\hline \multirow{3}{*}{$\begin{array}{c}\text { Inalteradas, } \\
\text { secado a } \\
\text { la sombra } \\
\text { durante } 10 \\
\text { días }\end{array}$} & N6 & 7.65 & 20 & 2.37 & 350 & 0.98 & $0.092^{1}$ & 10.7 \\
\hline & N7 & 8.53 & 22 & 2.00 & 400 & 1.10 & $0.032^{1}$ & 34.0 \\
\hline & NI1 & 9.32 & 36 & 1.75 & 700 & 1.27 & $0.039^{2}$ & 32.5 \\
\hline \multirow{2}{*}{$\begin{array}{c}\text { Inalteradas, } \\
\text { contenido } \\
\text { de humedad } \\
\text { natural }\end{array}$} & NI2 & 8.24 & 63 & 2.12 & 700 & 1.28 & $0.035^{2}$ & 37.1 \\
\hline & NI3 & 6.28 & 62 & 3.05 & 650 & 2.04 & $0.076^{2}$ & 26.8 \\
\hline \multirow{3}{*}{$\begin{array}{c}\text { Remoldea- } \\
\text { das }\end{array}$} & R1 & 10.59 & 35 & 1.41 & - & - & - & - \\
\hline & $\mathrm{R} 2$ & 7.65 & 43 & 2.34 & - & - & - & - \\
\hline & R3 & 6.28 & 43 & 3.07 & - & - & - & - \\
\hline
\end{tabular}

En la Tabla 2 se presentan, para cada muestra, la densidad seca inicial $\gamma_{d}$, la humedad natural $w$ y el índice de vacíos inicial $e_{0}$. Las muestras inalteradas en condición natural presentaron una densidad seca de 6.28 a $7.46 \mathrm{kN} / \mathrm{m}^{3}$, y humedad de 62 a 93\% (Figura 5). Tres muestras inalteradas 
fueron secadas a la sombra durante 10 días (muestras N6, N7 y NI1 en la Figura 5), alcanzando contenidos de humedad de 20 a $36 \%$ y densidades secas de 7.65 a $9.32 \mathrm{kN} / \mathrm{m}^{3}$.

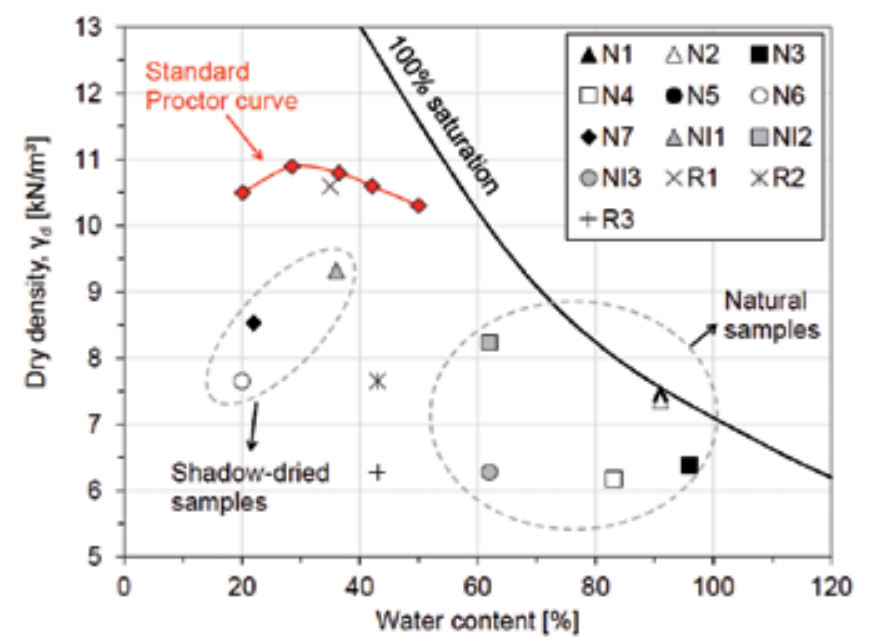

Figura 5: Resultados del ensayo Proctor Estándar y humedad/ densidad inicial en las muestras ensayadas

La Figura 6 muestra las curvas de compresión edométrica e isotrópica obteniendo una tensión de preconsolidación $\sigma_{c}^{\prime}$ promedio de $540 \mathrm{kPa}$ mediante el método de Casagrande $\left(\sigma_{v}^{\prime}\right.$ es la tensión vertical efectiva para los ensayos edométrico y $p^{\prime}$ es la presión de confinamiento efectiva para los casos isotrópicos). Considerando que la profundidad de extracción de las muestras no sobrepesa los $3 \mathrm{~m}$, el valor de la presión de preconsolidación es significativamente mayor que la presión in-situ; por lo tanto, la aparente preconsolidación del material debería explicarse mediante procesos de diagénesis (Hong et al., 2006; Sánchez, 2002).

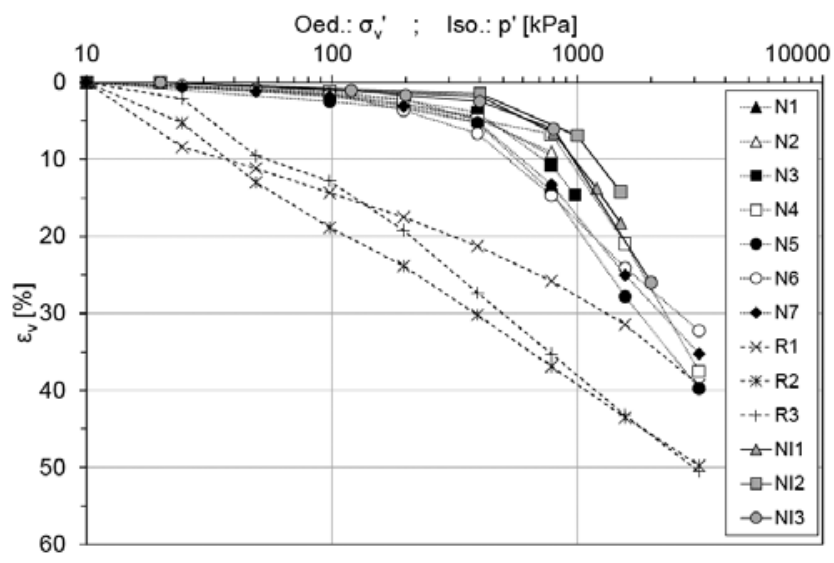

Figura 6: Curvas de consolidación edométrica e isotrópica

Los valores de $C_{s}$ y $C_{c}\left(\Delta e / \Delta \log \sigma^{\prime}\right.$, registrados a 24 horas bajo carga constante) son presentados en la Tabla 2, obteniendo un promedio en muestras inalteradas de 0.079 a)

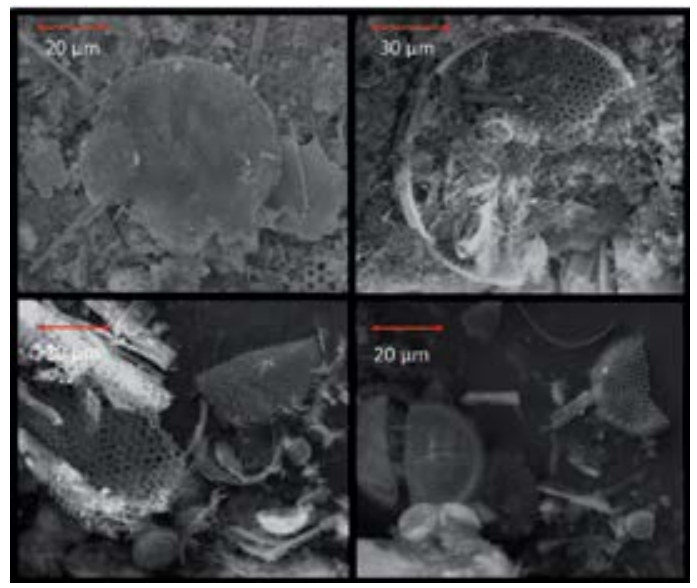

b)

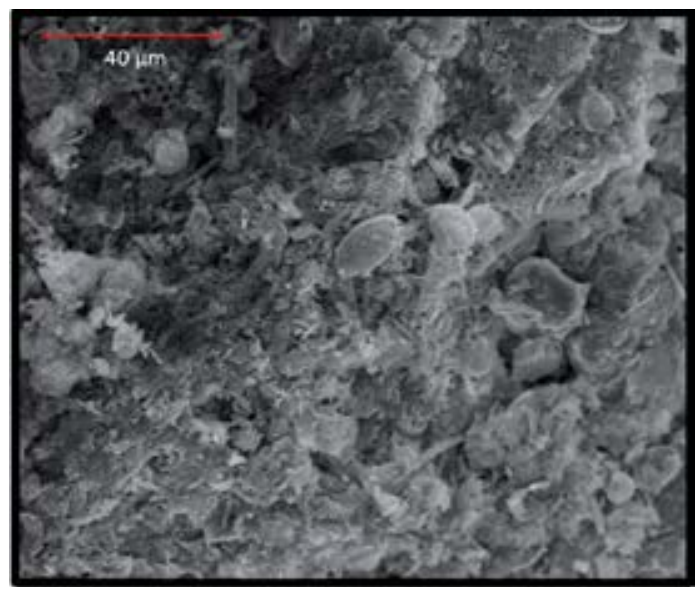

Figura 7: a) y b) Imágenes que muestran frústulas fracturadas encontradas en muestras post ensayo de consolidación

y 0.75 , respectivamente. Basado en diversos resultados empíricos, Terzaghi y Peck (1967) propusieron la relación empírica para el índice de compresión $C_{c}=0.009$ ( $L L[\%]-$ 13 ); usando $L L=62 \%$ para el suelo estudiado en este trabajo se obtiene un valor de $C_{c}=0.441$, lo que resulta significativamente más bajo comparado con lo obtenido experimentalmente. Conclusiones similares se obtienen al utilizar las correlaciones empíricas de Kulhawy y Mayne (1990).

La alta compresibilidad del suelo diatomáceo se debería fundamentalmente a que, sobre la presión de preconsolidación, ocurre la fractura de las frústulas de diatomea, generándose importantes deformaciones irreversibles por colapso de la estructura del suelo. Esto puede evidenciarse en observaciones de microscopio SEM de muestras post ensayo de consolidación mostradas en la Figura 7, donde se observa claramente que las frústulas se encuentran masivamente fracturadas. La rotura de frústulas ocasiona una reorganización de la estructura del suelo a un estado más denso bajo tensión constante, 
concordando con las observaciones microscópicas obtenidas por otros autores (e.g. Hong et al., 2006). Así, el comportamiento mecánico del suelo diatomáceo sería similar al de materiales granulares de granulometría relativamente uniforme en los que se supera la tensión de fluencia que gatilla la rotura de granos, generando grandes deformaciones plásticas (Biarez y Hicher, 1994; Mesri y Vardhanabhuti, 2009; Ovalle et al., 2015; Ovalle, 2013; Dano et al., 2017).

Más adelante, en la Figura 11 se presenta la evolución de $C_{c}$ (a 24 horas) en función de cada incremento de carga en muestras inalteradas. Se aprecia que este índice evoluciona hacia un valor constante de $C_{c}=1.0$ a 2.0 , siguiendo una tendencia análoga en comparación con la arena carbonatada (sensible a la rotura de granos) ensayada por Mesri y Vardhanabhuti (2009). Por otro lado, las muestras remoldeadas no presentan un cambio repentino ni brusco en compresibilidad ya que el proceso previo de remoldeo podría haber fracturado una cantidad significativa de diatomeas, generando una estructura interna estable.

\section{Consolidación secundaria}

Con el fin de estimar el término de la consolidación primaria, se monitoreó el exceso de presión de poros $\Delta u$ durante ensayos de consolidación isotrópica, disipándose en un $95 \%$ en aproximadamente $60 \mathrm{~min}$ (después de un incremento efectivo de 1000 a $1500 \mathrm{kPa}$; ver Figura 12). Se obtuvieron resultados similares en otros ensayos isotrópicos, por lo que es razonable asumir que la consolidación secundaria comienza después de 1 hora bajo carga constante. En la Figura 8 se presentan las curvas de consolidación en el tiempo para una muestra inalterada y remoldeada (los resultados son análogos para el resto de las muestras). De esta figura, se aprecia que en muestras inalteradas el efecto de creep aumenta significativamente con el nivel de tensión aplicado, particularmente para valores mayores a la presión de preconsolidación.

Como se ilustra en la Figura 9, se observan deformaciones considerables de creep para una muestra inalterada (resultados similares para otras muestras). La Figura 10 muestra el incremento de deformación por creep respecto a la curva de consolidación a 24 horas en diferentes tiempos de consolidación ( 5 a 50 días), donde se observa que la tasa de deformación aumenta significativamente después de la presión de preconsolidación del material, llegando incluso a diferencias volumétricas de $3 \%$ entre 1 y 50 días de carga constante.
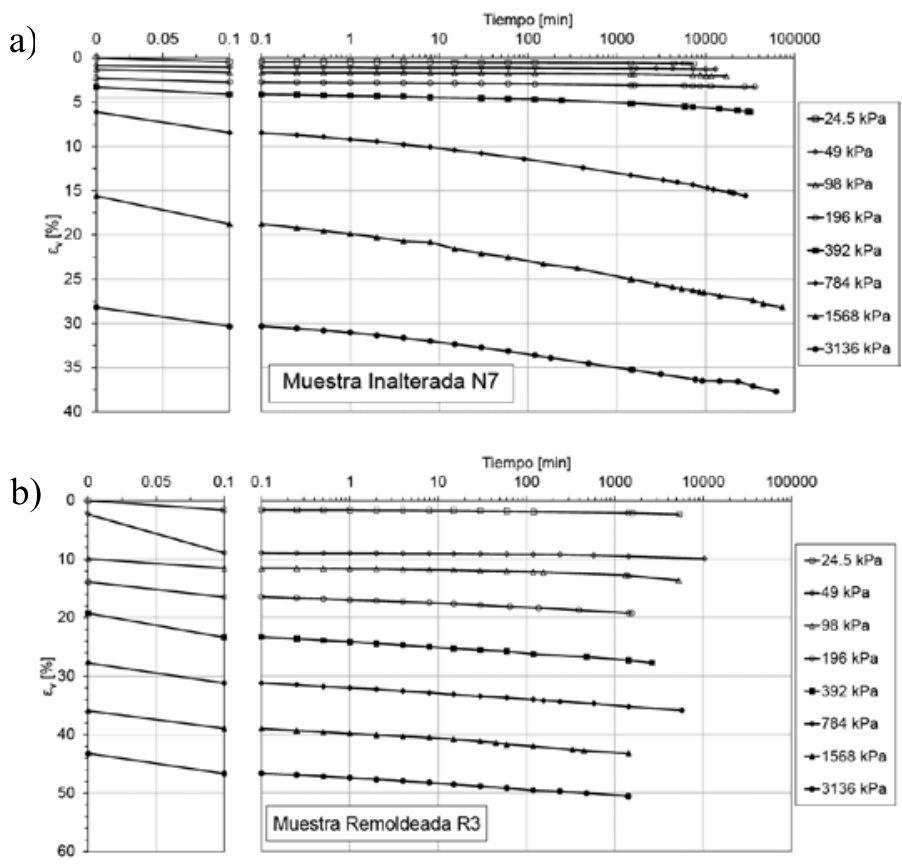

Figura 8: Tiempo-consolidación en muestra: (a) inalterada y (b) remoldeada

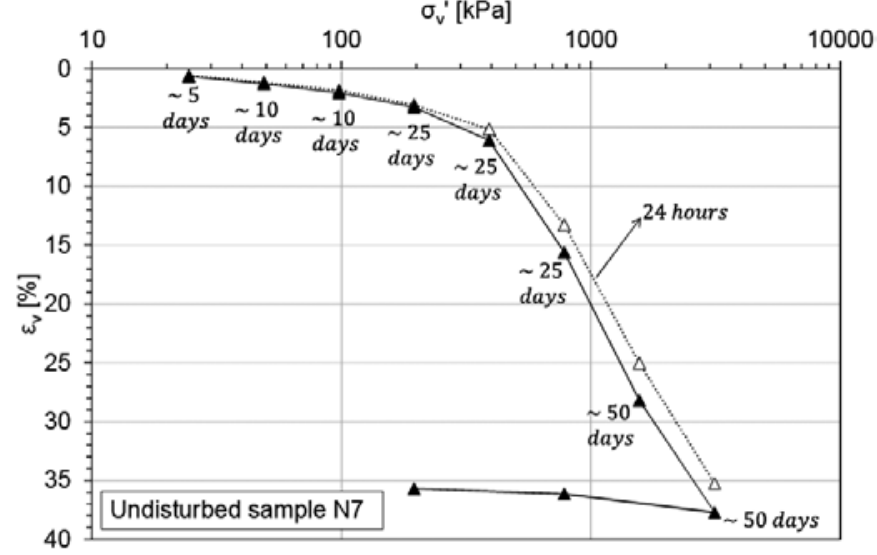

Figura 9: Consolidación después de 24 horas y 5 a 50 días (muestra N7)

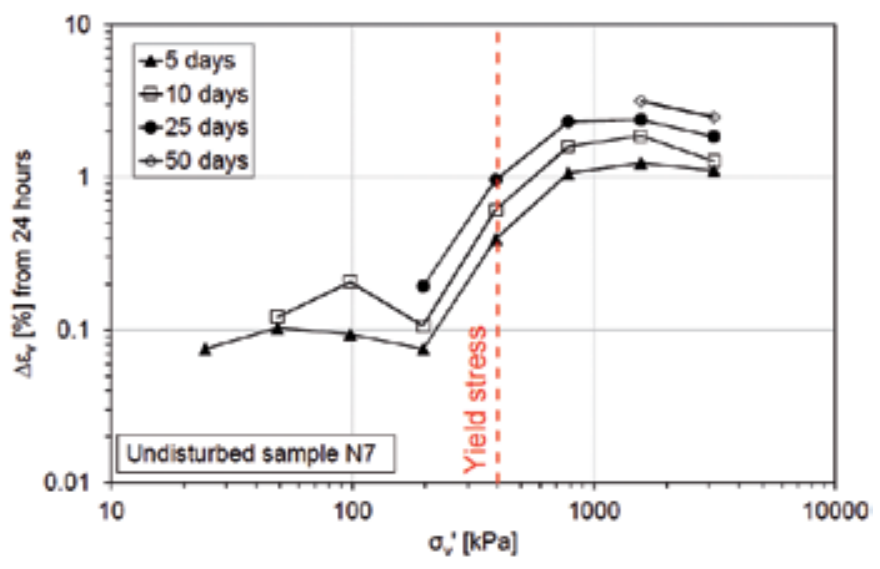

Figura 10. Incremento en la deformación por creep comparado con curva de consolidación a 24 horas (muestra N7) 
La evolución del creep se analizó a través del coeficiente de consolidación secundaria $C_{\alpha}=\Delta e / \Delta \log t$, que resulta prácticamente constante a partir de 1 hora bajo carga constante (Figura 8). La Figura 13 muestra que la evolución de $C_{\alpha}$ respecto a $\sigma_{v}^{\prime}$ en ensayos edométricos es equivalente a la tendencia de $C_{c}$ versus $\sigma_{v}^{\prime}$ (Figura 11); i.e. incrementa con $\sigma_{v}^{\prime}$ hasta un valor constante después de la presión de preconsolidación. Los resultados indican que se obtiene una razón constante $C_{\alpha} / C_{c}=0.042$, como se observa en la Figura 14, independiente del nivel de presión y tipo de muestra (inalteradas o remoldeadas). Lo anterior confirma lo propuesto por Mesri y Castro (1987), quienes propusieron que la relación $C_{\alpha} / C_{c}$ en un suelo es constante; para fines comparativos, valores reportados de $C_{\alpha} / C_{c}$ en arenas con rotura de partículas fluctúan en el orden de 0.015 a 0.030 (Mesri y Vardhanabhuti, 2009; Ovalle, 2018).

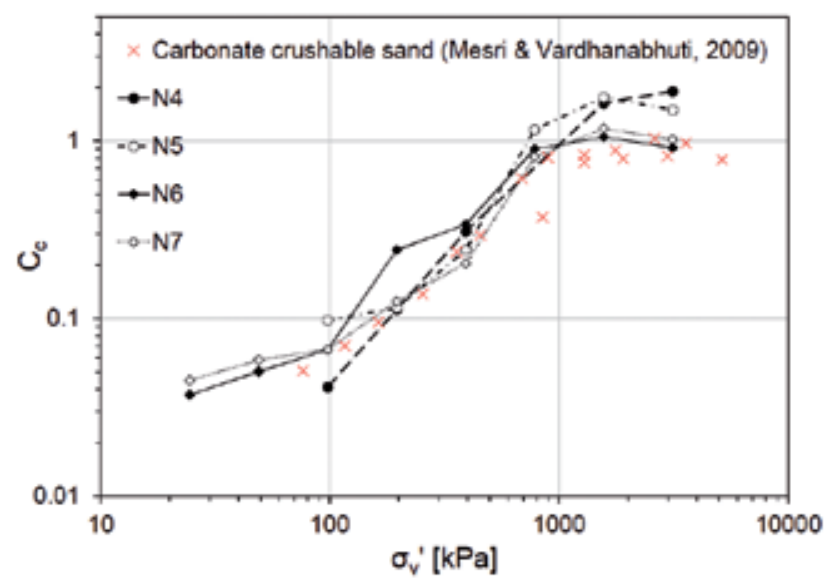

Figura 11: Evolución de $C_{c}$

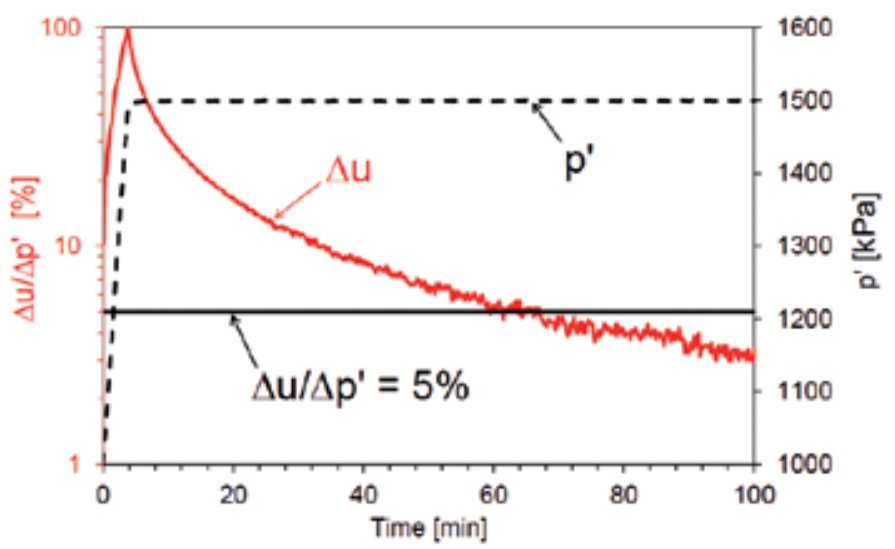

Figura 12: Evolución del exceso de presión de poros (muestra NI2)

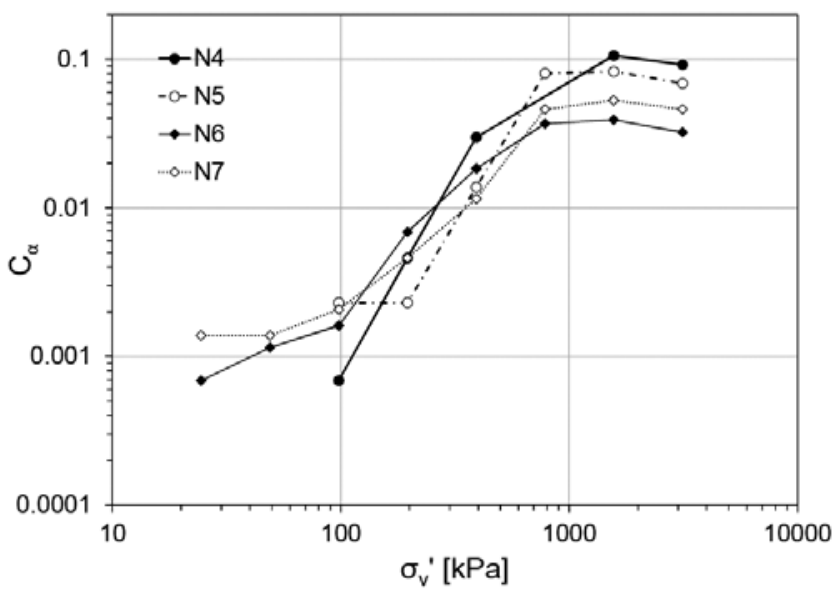

Figura 13: $C_{\alpha} / C_{c}$ en muestras inalteradas y remoldeadas

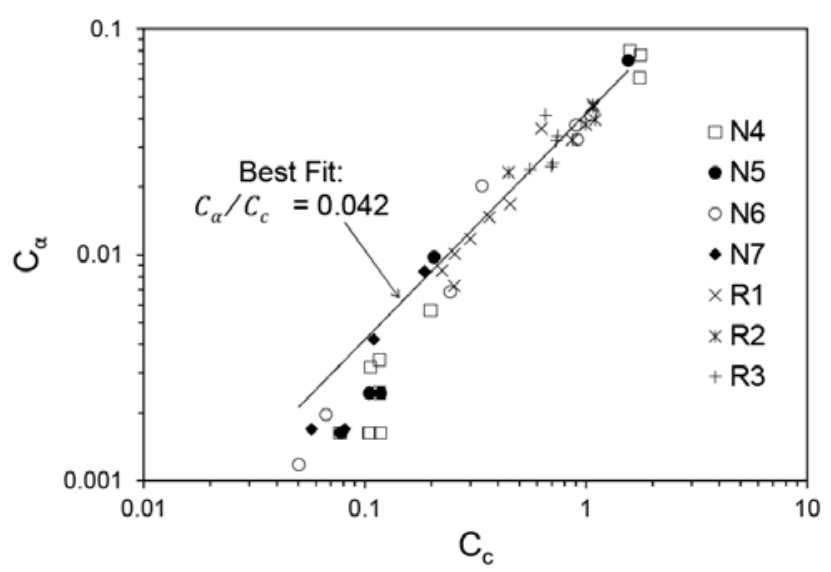

Figura 14: Evolución de $C_{\alpha}$

\section{Degradación del módulo de corte}

Se realizó una serie de ensayos de columna resonante $\mathrm{RC}$, torsión cíclica TC y triaxiales cíclicos en muestras inalteradas de $50 \mathrm{~mm}$ y $100 \mathrm{~mm}$ de altura a confinamientos de $100,200,400$ y $600 \mathrm{kPa}$, con saturación tal que $B>$ 95\%. En la Tabla 3 se presenta el resumen de los ensayos de RC y TC, donde $G_{\text {max }}$ y $V_{s}$ corresponden al módulo de corte máximo obtenido a la menor deformación de corte entregada por el ensayo (rango $\gamma=10^{-5}$ a $10^{-3} \%$ ), y a la velocidad de propagación de onda de corte respectivamente ( $V_{s}$ obtenido de la relación $G=\rho V_{s}^{2}$, con $\rho$ la densidad de masa del material). En la Tabla 4 se entrega el resumen de los ensayos triaxiales cíclicos, donde la determinación del módulo de corte $G$ se obtuvo asumiendo un coeficiente de Poisson $v=0.3 \pm 0.05$. En las Figuras 15 y 16 se presentan las curvas de degradación y amortiguamiento del material, donde $\gamma$ y $D$ corresponden a la distorsión angular y amortiguamiento, respectivamente. La Figura 17 muestra 
que los valores de $V_{s}$ obtenidos en este estudio están en el mismo rango que lo registrado por Maringue (2017) mediante métodos geofísicos in-situ.

La degradación de $G$ mostrada en la Figura 15 se observa a partir de una deformación de corte de $0.01 \%$, mientras que en curvas clásicas de la literatura para suelos finos (Vucetic y Dobry, 1991) y arenas (Seed e Idriss, 1970) esta tendencia comienza a $0.001 \%$. Por otro lado, a deformaciones superiores a $0.3 \%$ el suelo diatomáceo se asemeja a las curvas de degradación de suelos finos plásticos de $I P=15$ a 30. La degradación del material no se ve afectada por el confinamiento efectivo, lo que se asemeja a lo esperado en suelos finos (Ishibashi y Zhang, 1993).

Tabla 3: Resumen ensayos de columna resonante y torsión cíclica

\begin{tabular}{|c|c|c|c|c|c|c|}
\hline Muestra - Ensayo & $p^{\prime}, \mathrm{kPa}$ & $\begin{array}{c}\gamma_{\mathrm{d}}, \\
\mathrm{kN} / \mathrm{m}^{3}\end{array}$ & $e_{0}$ & $w, \%$ & $\begin{array}{c}G_{\max }, \\
\mathrm{MPa}\end{array}$ & $V_{s}, \mathrm{~m} / \mathrm{s}$ \\
\hline $1-\mathrm{RC}$ & 100 & 7.89 & 2.3 & 47.9 & 57 & 268 \\
\hline $1-\mathrm{TC}$ & 100 & 7.89 & 2.3 & 47.9 & 53 & 259 \\
\hline $1-\mathrm{RC}$ & 200 & 7.89 & 2.3 & 47.9 & 66 & 288 \\
\hline $1-\mathrm{TC}$ & 200 & 7.89 & 2.3 & 47.9 & 66 & 289 \\
\hline $2-\mathrm{RC}$ & 200 & 10.16 & 1.6 & 47.9 & 102 & 360 \\
\hline $2-\mathrm{TC}$ & 200 & 10.16 & 1.6 & 42.5 & 110 & 328 \\
\hline $2-\mathrm{RC}$ & 400 & 10.16 & 1.6 & 42.5 & 148 & 381 \\
\hline $2-\mathrm{TC}$ & 400 & 10.16 & 1.6 & 42.5 & 156 & 391 \\
\hline $2-\mathrm{RC}$ & 600 & 10.16 & 1.6 & 42.5 & 193 & 435 \\
\hline $2-\mathrm{TC}$ & 600 & 10.16 & 1.6 & 42.5 & 188 & 430 \\
\hline
\end{tabular}

Tabla 4: Resumen ensayos triaxiales cíclicos

\begin{tabular}{|c|c|c|c|c|c|c|c|c|}
\hline Muestra & $C_{\text {CSR }}$ tx & $\begin{array}{c}p^{\prime}, \\
\mathrm{kPa}\end{array}$ & $\begin{array}{c}\gamma_{\mathrm{d}}, \\
\mathrm{kN} / \mathrm{m}^{3}\end{array}$ & $e_{0}$ & $w, \%$ & $\gamma, \%$ & $G, \mathrm{MPa}$ & $D, \%$ \\
\hline 1 & 0.1 & 300 & 10.04 & 1.6 & 42.7 & 0.08 & $56 \pm 2$ & 4.6 \\
\hline 1 & 0.2 & 300 & 10.04 & 1.6 & 42.7 & 0.17 & $50 \pm 2$ & 6.3 \\
\hline 1 & 0.3 & 300 & 10.04 & 1.6 & 42.7 & 0.28 & $44 \pm 2$ & 7.6 \\
\hline 1 & 0.4 & 300 & 10.04 & 1.6 & 42.7 & 0.45 & $38 \pm 2$ & 9.5 \\
\hline 2 & 0.1 & 650 & 11.11 & 1.4 & 29.8 & 0.17 & $64 \pm 2$ & 2.3 \\
\hline 2 & 0.2 & 650 & 11.11 & 1.4 & 29.8 & 0.41 & $50 \pm 2$ & 3.7 \\
\hline 2 & 0.3 & 650 & 11.11 & 1.4 & 29.8 & 0.82 & $37 \pm 2$ & 5.2 \\
\hline
\end{tabular}

\section{Conclusiones}

Observaciones microscópicas SEM demuestran que el suelo diatomáceo de Mejillones contiene gran cantidad de frústulas fosilizadas de diatomeas, de superficie altamente rugosa, formas variadas y material silíceo. Estas partículas serían las responsables de la alta porosidad y humedad del suelo en condición natural. Asimismo, el agua retenida en las frústulas daría al suelo un alto Límite Líquido, pero no necesariamente asociado a una alta plasticidad

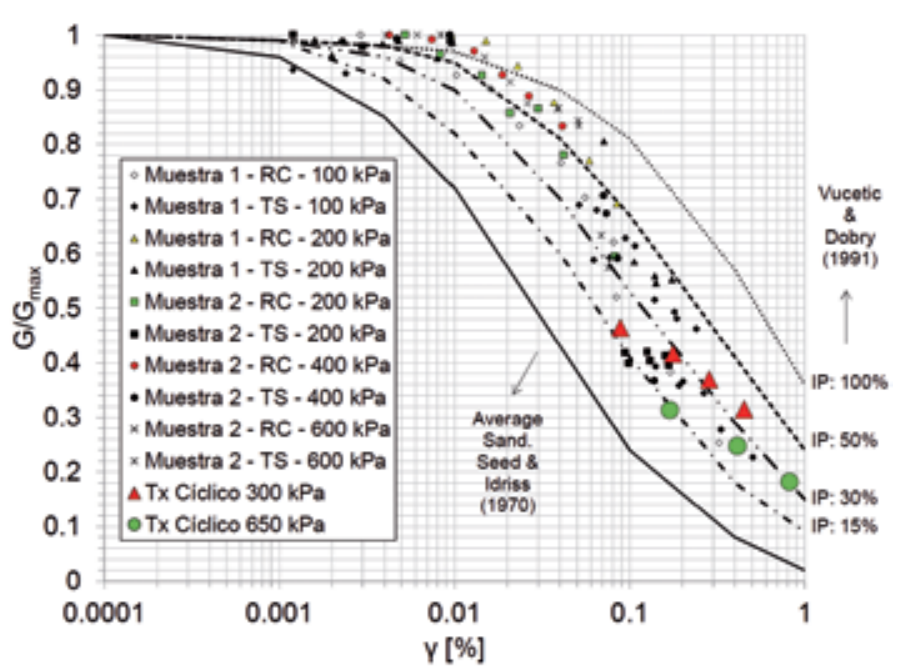

Figura 15: Curvas de degradación del módulo de corte normalizada

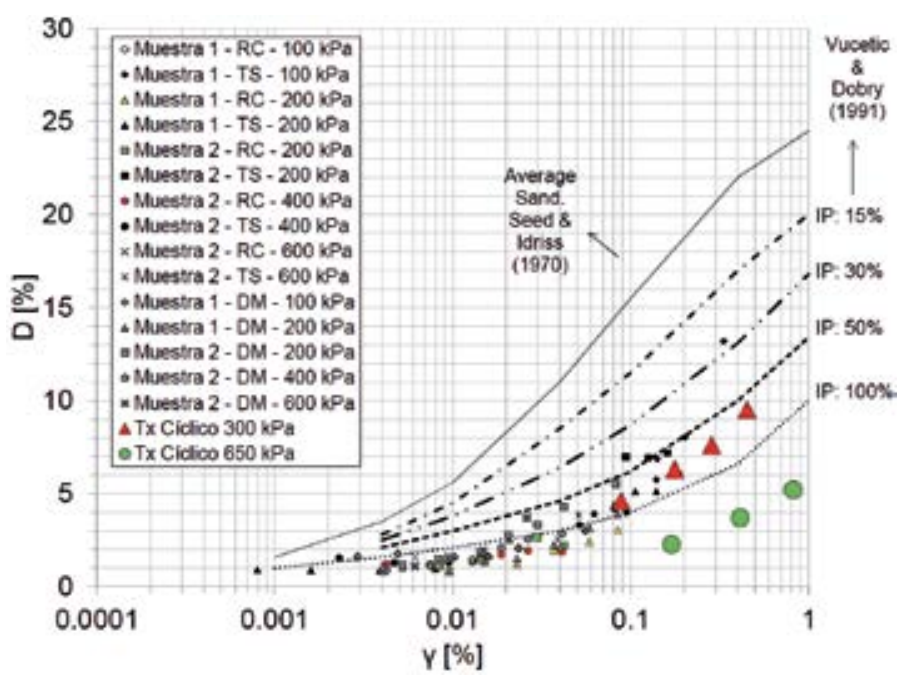

Figura 16: Curvas de amortiguamiento del material (DM: obtenido mediante decremento logarítmico)

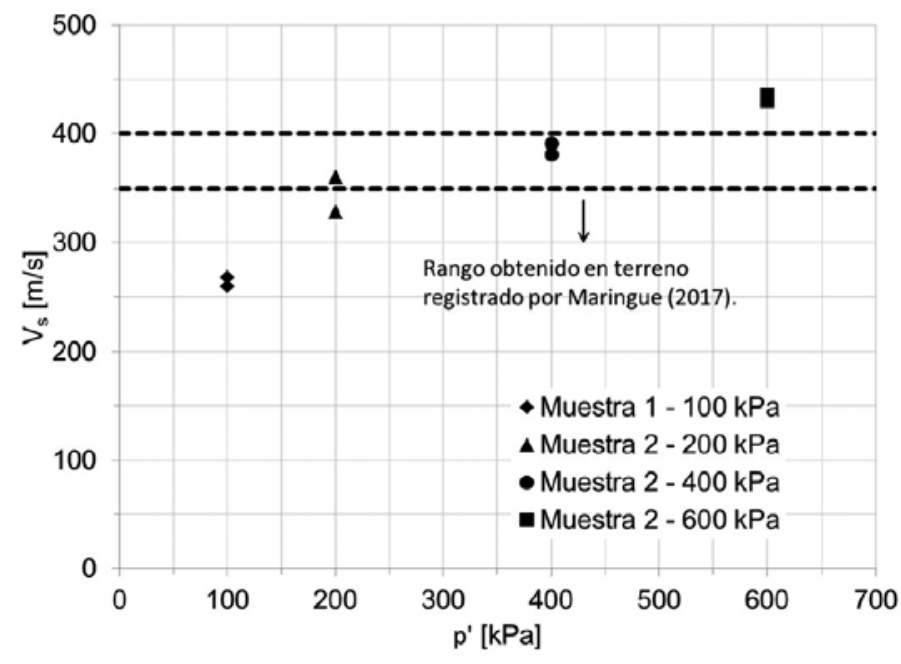

Figura 17: Curvas de degradación del módulo de corte normalizada 
en el contexto típicamente entendido en Ingeniería Geotécnica. Debido a las propiedades singulares dadas por las frústulas de diatomea, las correlaciones empíricas clásicas de la literatura para suelos finos no coinciden con el comportamiento de suelos diatomáceos, en especial aquéllas asociadas al Índice de Plasticidad .

A partir de ensayos de consolidación edométrica e isotrópica se obtuvo un índice de compresibilidad promedio $C_{c}$ de 1.17 y una presión de preconsolidación promedio de 540 $\mathrm{kPa}$. La preconsolidación estaría asociada a procesos de diagénesis puesto que el material estudiado fue extraído a una profundad no mayor de $3 \mathrm{~m}$. La alta compresibilidad se explicaría por la rotura de frústulas, como se observa en imágenes microscópicas en muestras post ensayo. Además, para cargas superiores a la presión de preconsolidación, el efecto del creep es considerable, con deformación volumétrica de hasta $3 \%$ a carga constante entre 1 y 50 días.

La curva de degradación cíclica del módulo de corte prácticamente no se ve afectada por el confinamiento efectivo, es significativamente rígida $\left(0.98 G_{\max }\right)$ hasta deformaciones del orden de $0.01 \%$, y no concuerda con las correlaciones empíricas para suelos finos. Posterior a este valor de deformación, el material se degrada alcanzando valores típicamente asociados a de suelos finos. Los valores de $G_{\max }$ son consistentes con resultados de $V_{s}$ obtenidos in-situ.

\section{Agradecimientos}

Este estudio se enmarca dentro del proyecto FONDECYT 11150084, financiado por CONICYT Chile. Los autores agradecen el aporte de Petrus Geotécnicos Consultores Ltda. en la realización de este trabajo.

\section{Referencias}

Arenaldi, G. (2018). Caracterización geotécnica de suelos diatomáceos. Tesis de Magíster en Ciencias de la Ingeniería, Pontificia Universidad Católica de Chile

ASTM D2435 (2011). Standard test method for one-dimensional consolidation properties of soils using incremental loading. West Conshohocken, PA, USA, ASTM International

ASTM D4767 (2011). Standard test method for consolidated undrained triaxial compression test for cohesive soils. West Conshohocken, PA, ASTM International
Biarez, J. and Hicher, P.Y. (1994). Elementary mechanics of soil behaviour: saturated remoulded soils. Balkema, The Netherlands Caicedo, B., Mendoza, C., López, F. and Lizcano, A. (2018). Behavior of diatomaceous soil in lacustrine deposits of Bogotá, Colombia. Journal of Rock Mechanics and Geotechnical Engineering 10(2), 367-379.

Dano, C., Ovalle, C., Yin, Z., Daouadji, A. and Hicher, P.Y. (2017). Behavior of granular materials affected by grain breakage. Advances in Multi-Physics and Multi-Scale Couplings in Geo-Environmental Mechanics, 1st Edition. Eds. Nicot, F. and Millet, ISTE Press, 95-132

Díaz-Rodríguez, J.A. and González-Rodríguez, R. (2013). Influence of diatom microfossils on soil compressibility. $18^{\text {th }}$ International Conference on Soil Mechanics and Geotechnical Engineering, Paris, 325-328

González, J. (2013). Geología y estructura submarina de la Bahía de Mejillones: su vinculación con la deformación activa en la plataforma emergida a los $23^{\circ} \mathrm{S}$. Memoria para optar al título de Geólogo, Universidad Católica del Norte, Antofagasta, Chile

Hong, Z., Tateishi, Y. and Han, J. (2006). Experimental study of macro-and microbehavior of natural diatomite. Journal of Geotechnical and Geoenvironmental Engineering 132(5), 603-610

Ishibashi, I. and Zhang, X. (1993). Unified dynamic shear moduli and damping ratios of sand and clay. Soils and Foundations 33(1), 182-191

Kulhawy, F.H. and Mayne, P.W. (1990). Manual on estimating soil properties for foundation design. Geotechnical Engineering Group

Maringue, J. (2017). Amenaza sísmica en Mejillones mediante una aproximación geofísica: análisis dinámico y amplificación sísmica asociada. Tesis de Magíster en Ciencias de la Ingeniería, Pontificia Universidad Católica de Chile, Santiago, Chile

Mesri, G. and Vardhanabhuti, B. (2009). Compression of granular materials. Canadian Geotechnical Journal 46(4), 369-392

Mesri, G. and Castro, A. (1987). $\mathrm{C}_{\alpha} / \mathrm{C}_{\mathrm{c}}$ concept and $\mathrm{K}_{0}$ during secondary compression. Journal of Geotechnical Engineering 113(3), 230-247

Muñoz, E., y Rivas, M. (2012). Modificación plan regulador comunal de Mejillones, zona urbana consolidada y portuaria Mejillones. Gobierno de Chile 
Nazar, R., Ovalle, C., Barrios, A. y Arenaldi, G. (2016). Caracterización geotécnica y resistencia al corte de suelos diatomáceos y de la interfaz suelo-acero. IX Congreso Chileno de Ingeniería Geotécnica, Valdivia

Nava, T. (2007). Influencia de las diatomeas en la resistencia al corte del caolín. Tesis de Maestría, Universidad Nacional Autónoma de México, México

Ovalle, C. (2018). Role of particle breakage in primary and secondary compression of wet and dry sand. Géotechnique Letters 8(2), 161-164

Ovalle (2013). Contribution à l'étude de la rupture des grains dans les matériaux granulaires. Doctoral thesis, Ecole Centrale de Nantes, France.

Ovalle, C., Dano, C., Hicher, P.-Y. and Cisternas, M. (2015). Experimental framework for evaluating the mechanical behavior of dry and wet crushable granular materials based on the particle breakage ratio. Canadian Geotechnical Journal 52(5), 587-598

Sánchez, M.A. (2002). Caracterización geomecánica de diatomeas. Tesis para optar al título de Ingeniero Civil, Universidad de Chile, Santiago, Chile

Seed, H.B. and Idriss, I.M. (1970). Soil moduli and damping factors for dynamic response analysis. Earthquake Engineering Research Center, University of California, Berkeley, California, USA
Shiwakoti, D.R., Tanaka, H., Tanaka, M. and Locat, J. (2002). Influences of diatom microfossils on engineering properties of soils. Soils and Foundations 42(3), 1-17

Terzaghi, K. and Peck, R.B. (1967). Soil Mechanics in Engineering Practice. John Wiley \& Sons, New York

Treguer, P., Nelson, D.M., Van Bennekom, A.J., DeMaster, D.J., Leynaert, A. and Quéguiner, B. (1995). The silica balance in the world ocean: a reestimate. Science 268(5209), 375-379

Verdugo, R. (2008). Singularities of geotechnical properties of complex soils in seismic regions. Journal of Geotechnical and Geoenvironmental Engineering 134(7), 982-991

Vucetic, M. and Dobry, R. (1991). Effect of soil plasticity on cyclic response. Journal of Geotechnical Engineering 117(1), 89-107

Wesley, L. D. (2010). Discussion of "Singularities of geotechnical properties of complex soils in seismic regions" by Ramon Verdugo. Journal of Geotechnical and Geoenvironmental Engineering 136(1), 277-279 